\title{
Visual cognition: a new look at the two-visual systems model
}

\author{
M. Jeannerod ${ }^{\mathrm{a}, *}$, P. Jacob $^{\mathrm{b}}$ \\ a Institut des Sciences Cognitives, Lyon, France \\ b Institut Jean Nicod, Paris, France
}

Abstract

In this paper, we argue that no valid comparison between visual representations can arise unless provision is made for three critical properties: their direction of fit, their direction of causation and the level of their conceptual content. The conceptual content in turn is a function of the level of processing. Representations arising from earlier stages of processing of visual input have very little or no conceptual content. Higher order representations get their conceptual content from the connections between visual cognition and other parts of the human cognitive system. The two other critical properties of visual representations are their mind/world direction of fit and their mind/world direction of causation. The output of the semantic processing of visual input has a full mind-to-world direction of fit and a full world-to-mind direction of causation: it visually registers the way the world is and is caused by what it represents. The output of the pragmatic processing yields information for the benefit of intentions, which clearly have a world-to-mind direction of fit and a mind-to-world direction of causation. An intention is both the representation of a goal and a cause of the transformation of a goal into a fact. These properties segregate representations specialized for perception from those specialized for action. Perception implies comparison between simultaneously represented and analyzed objects: hence, object perception presupposes the representation of spatial relationships among objects in a coordinate system independent from the perceiver. Spatial relationships carry cues for attributing meaning to an object, so that their processing is actually part of semantic processing of visual information. These considerations lead to a re-evaluation of the role of the two classical pathways of the human visual system: the ventral and the dorsal cortical pathways. The parietal lobe, which has been identified with the dorsal pathway, cannot be considered as a unitary entity with a single function. The superior parietal lobule carries visuomotor processing, a non-lateralized process. The right inferior parietal lobule contributes to the perception of spatial relationships, a process with a mind-to-world direction of fit and a world-to-mind direction of causation. Finally, the left inferior parietal lobule contributes to still another type of representation, related to visually goal-directed action, i.e., with both a world-to-mind direction of fit and a mind-to-world direction of causation.

(C) 2004 Published by Elsevier Ltd.

Keywords: Visual cognition; Two-visual systems hypothesis; Representations; Goal-directed movements; Mental imagery

\section{Introduction: the emergence of the two-visual systems hypothesis}

Although seeing is commonly experienced as a unitary activity, the scientific understanding of human vision resists such a simple view. Both psychologists and neuroscientists consider that the processing of visual information is distributed across several different routes which eventually reach different functional outcomes, and that these processing routes can be mapped onto well-identified anatomical subdivisions of the visual system. This general idea finds support

* Corresponding author. Tel.: +33 3791 1212; fax: +33 37911210.

E-mail address: jeannerod@isc.cnrs.fr (M. Jeannerod). in the anatomical organization of the visual system in all the vertebrate species that have been studied over the last hundred years, including frogs, fishes, cats, rats, bats, tree-shrews or monkeys, where the retina projects onto many different cortical and subcortical relays. Indeed, the early versions of the two-visual systems hypothesis were first entertained by neurophysiologists working on the visual systems of non-human animals. In amphibians, for example, it was demonstrated by Ingle (1973) that prey-catching behavior is mediated by retinal projections onto the optic tectum, while the visual control of barrier-avoidance is mediated by retinal projections onto pretectal nuclei. Similarly for mammalians, it was demonstrated by Schneider (1969) that a hamster with a lesioned superior colliculus could discriminate vertical from horizon- 
tal stripes but could not run a maze. Conversely, a hamster with a lesioned visual cortex could run a maze but not do pattern recognition. Since the earlier evidence came from the study of animals with little or no visual cortex, early versions of the two-visual systems hypothesis emphasized the contrast between vision controlled by peripheral retinal information, based on subcortical structures, and vision based on cortical structures, respectively.

The first major step was taken by Ungerleider and Mishkin (1982), who located the two-visual systems within the primate visual cortex. They examined the selective effects of lesions in the brains of macaque monkeys on two kinds of behavioral tasks: a landmark task and an object-discrimination task. In the former task, the monkey had to discriminate between two covered wells-one empty and one containing a reward-according to whether they were located far away or near a landmark. In the latter task, the monkey had to discriminate two objects of different shapes, colors and textures. Ungerleider and Mishkin found that a lesion in the inferotemporal cortex severely impaired the animal in the object-discrimination task, but not in the landmark task. Conversely, they found that a lesion in the posterior parietal cortex severely affected the animal's performance in the landmark task, but not in the object-discrimination task. On the basis of these experiments, Ungerleider and Mishkin concluded that both the ventral stream (which they called the 'object-channel') and the dorsal stream (which they called the 'space-channel') were specialized in perceiving different aspects of the visual world. Indeed, their landmark task tested the animal's ability to perceive spatial relations, not to act on a target.

The second major step was taken by Milner and Goodale (1995) when they provided room for the visuomotor transformation within their amended version of the two-visual systems model of human vision. The visuomotor transformation is the automatic conversion of visual information into hand commands for reaching and grasping objects-a topic whose study was pioneered in the monkey by Mountcastle and collaborators in the mid-1970s. In Milner and Goodale's view, the ventral stream underlies what they call 'vision-forperception' and the dorsal stream underlies what they call 'vision-for-action'. The crucial evidence on which Goodale and Milner based their revised interpretation of the two-visual systems model of human vision is the neuropsychological double dissociation between two visual impairments produced by two selective lesions in the human visual system: a form of visual agnosia resulting from lesions in the inferotemporal area and optic ataxia resulting from lesions in the posterior parietal cortex. Visual agnosic patients are deeply impaired in the visual recognition of the color, size, shape and orientation of objects. But they can reach and grasp objects they cannot visually recognize. Conversely, optic ataxic patients fail to reach and grasp objects whose shapes, sizes and orientations they can visually recognize.

Many relevant psychophysical experiments have confirmed the view that one and the same visual stimulus can be processed in two different ways according to the task by healthy human subjects. Consider, for example, the common illusion of visual 'induced motion': a small stationary visual target is presented on a screen against a large background of dots constantly moving in one direction. A subject located in front of the screen will report that the small target appears to be moving in the direction opposite to the background. If, however, the subject is instructed to point at the small target (with his unseen hand in order to avoid visual guidance of the pointing movement), he will accurately reach the actual position of the target. As this example (Bridgeman, Kirsch, \& Sperling, 1981) shows, visual perception and visually guided action can be dissociated by carefully designed experiments in normal subjects.

Thus, by the mid-1990s, the two major versions of the two-visual systems model of human vision disagreed on the functional significance of the dorsal pathway and the role of the posterior parietal lobe. Ungerleider and Mishkin's (1982) model subscribes to the assumption that the major function of the primate visual system is visual perception: the two cortico-cortical pathways in the primate visual brain underlie perceptual awareness. By contrast, according to Milner and Goodale's (1995) model, perceptual awareness is not the exclusive (or the main) function of vision in primates. Corticocortical pathways in the primate and the human brains are not limited to visual perception.

We do accept Milner and Goodale's (1995) basic bifurcation between vision-for-perception and vision-for-action, which we call, respectively, the "semantic" and the "pragmatic" processing of visual information (see, e.g., Jeannerod, 1997). We shall argue that Milner and Goodale's model of the two-visual systems hypothesis seriously underestimates the complexity of the representations of actions produced by the pragmatic processing of visual information. No doubt, one of the functions of the dorsal stream is to enable the visuomotor transformation. But, as we shall argue in this paper, the human parietal lobe has two other major functions: one is to allow the perception of spatial relations among objects; the other is to store complex representations of actions (such as schemas for the use of cultural tools). On the perceptual (or semantic) side, it would be absurd to reduce the scope of human vision to the perception of objects that one can manipulate with one's hand. Humans can of course visually perceive a great variety of other things such as clouds, flames, shadows, holes and many others. In particular, humans can also visually perceive actions performed by conspecifics. Similarly, the visual control of human actions should not be restricted to the visuomotor transformation, i.e., to reaching and grasping objects. Humans can plan, execute and visually control far more complex actions.

\section{The architecture of visual cognition}

The basic insight of the two-visual systems hypothesis is that the goal of visual processing is two-fold: on one hand, hu- 
man visual perception is a rich source of knowledge about the world; on the other hand, human vision contributes to visually guided actions on the world. The computational requirements, respectively, of perception and the control of objectoriented actions on the human visual system are clearly different. Perception itself fulfills two complementary functions: selection and recognition. The selection phase consists in both segregating a complex visual array into several separable objects and in attributing to each separate object its own set of appropriate visual attributes (this is the so-called "binding" problem). Usually, the color and texture of an object will be highly relevant to its perceptual selection from a set of neighboring objects. Segregation and binding require that the relative spatial locations of different objects in a visual array be coded by the perceptual system. Since perceptual recognition of an object must be achieved from many different spatial perspectives on many different occasions, it requires encoding of visual information about an object's enduring properties. In other words, perceptual recognition of an object demands that visual information about a perceived object matches conceptual information and knowledge about it stored in long-term memory.

Once an object has been perceptually selected from a set of competitors, the visual control and monitoring of the action of prehension can take over. Whereas the color and texture of an object are relevant to its perceptual selection, they are not relevant to grasping it. What is relevant to the visual guidance of grasping an object are its absolute shape, size and orientation together with its position relative to the agent's body.

Two of the most fundamental dimensions along which visual percepts differ from visuomotor representations are what, following Anscombe (1957) and Searle (1983), philosophers of mind and perception call, respectively, their direction of fit and their direction of causation (for a full account of this distinction, see Jacob \& Jeannerod, 2003). Beliefs and visual percepts are descriptive representations. They have a mind-to-world direction of fit: their job is to represent facts or actual states of affairs. If what a belief or a percept represents fits a mind-independent fact in the world, then the belief or the percept is veridical; otherwise, they are not. By contrast, intentions and desires are prescriptive representations. They have a world-to-mind direction of fit: their job is to represent goals, i.e., possible or impossible (i.e., non-actual) states of affairs. If what obtains in the world fits what the intention or the desire represents, then the intention or the desire is fulfilled; otherwise, they are not.

In addition, percepts and intentions have an opposite mind-world direction of causation. If you perceive a basket full of apples, pears, lemons and oranges, your visual percept is caused by the state of affairs that it represents. Unless there was a basket full of apples, pears, lemons and oranges, you might hallucinate one, but you could not perceive it. Whereas visual percepts are caused by what they represent, intentions cause the state of affairs which they represent. When you intend to pick up an apple from the perceived basket full of fruits in front of you, you represent a goal for action, i.e., a possible non-actual state of affairs that consists of your holding a particular apple in your hand. When you form the intention to grasp the apple, the perceived fact is that the target of your action (i.e., the apple) is in the basket, not in your hand. Your intention causes your action which in turn causes a new fact to obtain, i.e., the apple's being in your hand.

Clearly, visual percepts and beliefs have the same mindto-world direction of fit. The formation of beliefs about the world is a step towards the acquisition of knowledge about the world. While beliefs have a conceptual content, visual percept has a rich pictorial non-conceptual content. The function of visual percepts is to provide visual information relevant to the formation of beliefs, and thus of knowledge about the visual properties of the world. The philosopher Millikan (1996) has argued that there exists, in the human mind (and in the minds of other animals), a class of Januslike mental representations, which she calls "pushmi-pullyu" representations-after the Pushmi-Pullyu, an imaginary twoheaded animal in Dr. Doolittle's stories. Visuomotor representations are such representations with a hybrid direction of fit in virtue of which they provide motor intentions, not beliefs, with visual information about affordances for action. Because they represent only immediate affordances for action, the non-conceptual content of visuomotor representations is not as rich as the non-conceptual content of visual percepts.

The contrast between the direction of fit, respectively, of visual percepts and visuomotor representations is confirmed by the neuropsychological double dissociation already mentioned between the perceptual impairment of visual agnosic patients and the visuomotor impairment of optic ataxic patients. The visual form agnosic patient DF cannot form visual percepts, but she can still form visuomotor representations of targets of hand actions (Goodale, Milner, Jakobson, \& Carey, 1991). Conversely, optic ataxic patients cannot form visuomotor representations of targets of hand action, but they can form visual percepts (Jeannerod, 1986).

\section{Levels of semantic processing of visual information}

What we call "semantic" processing of visual information is the process whereby visual inputs are transformed into perceptual representations with a mind-to-world direction of fit and whose pictorial non-conceptual contents must ultimately match the conceptual contents of beliefs. As we pointed out above, the goal of semantic processing of visual inputs is recognition of objects which involves segregation of a scene into separable objects and binding to each object of its appropriate visual attributes. Only representations with a fairly abstract conceptual content can be used in thinking and reasoning about objects. In order to match the conceptual contents of general thoughts and beliefs stored in memory, much of the detailed pictorial content of visual percepts must be selectively eliminated. 
At the lowest level, perception can be, as philosophers of perception (e.g., Dretske, 1969, 1978) call it, non-epistemic. Suppose you are driving very fast. You see something lying on the road ahead of you. You cannot identify it because you are moving too fast, but you nonetheless skillfully avoid hitting it. Unless you saw it, you would have hit it. So you did see it, but you could not see what it was. Your visual perception of the object on the road was non-epistemic. Epistemic visual perception involves further processing of an object giving rise to some identification: one sees epistemically, not just an object, but an object as instantiating some category or other. Alternatively, one sees epistemically the fact that an object falls under some concept or other. Seeing that the car in the street is moving at a slow speed, for example, is forming a visual percept of an object with a global contour and several parts of different shapes, colors and textures that move together relative to other surrounding objects. For the purpose of considering the functional advantages or disadvantages of, e.g., a Ford Mustang with cars of other brands, however, one must switch from the detailed pictorial representation of a visual percept to more general knowledge about cars sustained by representations with a more abstract conceptual content (e.g., the concept of a Ford Mustang).

Similarly, one cannot visually perceive a mug of beer as being to the left of a bottle of wine without representing, e.g., the particular shades of colors and the levels of the liquids contained in both the mug and the bottle, and the particular shapes of the mug and the bottle. Now, a mug of beer can only be seen to be to the left of a bottle of wine from some spatial point of view, e.g., from the point of view of someone facing the window, not from the point of view of someone with her back onto the window. From the latter point of view, one will see the same mug of beer to the right, not to the left, of the same bottle of wine. Now, one can think about, but one cannot currently see, the point of view by means of which one is currently seeing a mug of beer as being to the left of a bottle of wine. One may see at $t$ the point of view one occupied at $t-1$, by occupying at $t$ a point of view different from the one is currently seeing at $t$. So in order to form the thought that the mug of beer is to the left of the bottle of wine from the point of view of someone facing the window, not from the point of view of someone with his back onto the window, one must ascend from a visual percept with a rich pictorial non-conceptual content to a thought with a more abstract conceptual content. One must abstract away from the pictorial content of the visual percept representing, e.g., the colors and levels of the two liquids and the shapes of the mug and the bottle, in order to form a conceptual representation of the spatial relation " $x$ is to the left of $y$ from $z$ 's point of view".

Much of our knowledge about the mechanisms underlying visual recognition and identification of objects comes from the observation of patients with brain lesions. Clinical observation has provided information which could have hardly been obtained from studies on subjects with an intact brain. Specifically, clinical observation gives firm support to the idea of the existence of levels of perceptual processing of visual inputs. The higher levels of processing correspond to the common experience of seeing, which can be easily studied in normal subjects: this is the goal of cognitive perceptual psychology. By contrast, the lower levels, which correspond to covert stages of processing, become only apparent in patients where a lesion has impaired the higher levels.

Indeed, visual identification disorders resulting from lesions of the equivalent of the ventral pathway in the human visual system correspond to impairments of visual information processing at different levels. When they are bilateral, posterior lesions affecting the lateral occipital region destroy visual representations resulting from an early stage of perceptual processing (what philosophers call non-epistemic visual perception). Such representations have a rich informational content and little or no conceptual content. The resulting effect (visual form agnosia) is that patients with such lesions cannot form simple percepts from the visual array: they cannot recognize the simple shapes, orientation and color of visual stimuli. As a consequence of this impairment, the more cognitive visual representations (those with a conceptual content) are 'deafferented' from visual input and cannot achieve their task of object recognition.

Several observations, however, suggest that these higher representations may still be functional. Servos and Goodale (1995), for example, found that the visual form agnosic patient DF had retained the ability to form visual mental images of objects: although she could not recognize visually presented objects and could not draw copies of seen objects, she could draw copies of objects from memory-which she then could hardly recognize. Patients with even more posterior occipital lesions including lesions of the primary visual areas in the calcarine sulcus, who present the typical picture of cortical blindness, spontaneously report vivid visual images (and sometimes even deny being blind, see Goldenberg, Müllbacher, \& Nowak, 1995).

More anterior lesions (e.g., bilateral or predominantly leftsided lesions of the inferotemporal cortex) destroy more cognitive representations with conceptual content, those that give access to the meaning of the percepts, and allow processes like comparison and categorization. The resulting effect is "associative agnosia", a condition in which patients often retain the ability to identify simple shapes and are even able to copy line drawings of objects that they cannot recognize. Whereas presemantic recognition of objects is preserved in these patients (they are able to form visual percepts), full semantic identification seems to be lost. Not surprisingly, these patients are usually unable to perform mental visual imagery tasks (although there are several well-documented exceptions to this rule, see Berhmann, Winocur, \& Moscovitch, 1992). The loss of the ability to mentally image visual objects (e.g., faces) is congruent with the findings obtained with neuroimaging techniques from normal subjects during mental visual imagery tasks. Typically, these tasks activate brain areas at the occipitotemporal junction as well as in the inferotemporal cortex (see Farah, 1995): the activated ar- 
eas superimpose with those activated during recognition and matching of seen objects (e.g., Ungerleider \& Haxby, 1994). These results, together with the effects of anterior lesions of the ventral pathway, thus suggest that the inferotemporal cortex might be a critical site for semantic processing, including long-term declarative memory, of visual objects. The network for the generation of mental visual imagery of objects, as described with the use of neuroimaging techniques in normal subjects, also includes more posterior occipital areas, including areas in the calcarine sulcus (Kosslyn et al., 1993; Kosslyn, DiGirolamo, Thompson, \& Alpert, 1998). Although at first sight this result seems in conflict with preservation of the ability to evoke such images in patients with occipital lesions, it is possible that a more complete analysis of visual mental imagery in agnosic patients with posterior lesions will reveal subtle impairments with respect to the normal process.

The feed-forward mode of information processing is often considered as the main (if not the only) constituent of visual cognition. Indeed, in his own definition of visual cognition, Pinker (1985) states that it can be conveniently divided into two serially organized steps which indeed fulfill our criteria for a world-to-mind direction of fit. Pinker's first step "is the representation of information concerning the visual world currently before a person [...] the process that allows us to determine on the basis of retinal input that particular shapes, configurations of shapes, objects scenes and their properties are before us". The second step "is the process of remembering or reasoning about shapes or objects that are not currently before us but must be retrieved from memory or constructed from a description" (pp. 2-3). Whereas Pinker's first stage is consistent with the world-to-mind direction of causation we ascribed to visual percepts, Pinker's second stage is more like beliefs, which may well fail the world-to-mind direction of causation.

\section{Levels of pragmatic processing of visual information}

As we suggested in Sections 1 and 2, not all visual representations are percepts with a world-to-mind direction of fit. They do not all result from semantic processing. There are also visuomotor representations that result from the pragmatic processing of visual inputs. As we argued above, visuomotor representations have a hybrid direction of fit that makes them suitable for providing motor intentions with visual information about targets of action. Unlike percepts and beliefs, intentions have a mind-to-world direction of fit and unlike percepts, they have a mind-to-world direction of causation: they cause bodily movements that turn a possible into an actual state of affairs.

For the purpose of introducing the notion of pragmatic processing, we focused on visuomotor representations that are involved in the visuomotor transformation, i.e., in the visual control of reaching and grasping objects. What is crucial to the content of such visuomotor representations is that they represent the geometrical properties of objects relevant for grasping and that they code the spatial position of the target in egocentric coordinates, i.e., in a frame of reference centered on the agent's body. The visuomotor transformation is but the lowest level of pragmatic processing of visual inputs. Visuomotor representations of targets of prehension are representations with little or no conceptual content at all. The scope of pragmatic processing, however, is not limited to the visuomotor transformation, since pragmatic processing is involved in conceptually more complex operations like evaluating the feasibility of an action, anticipating its consequences, planning further steps and learning the skilled use of tools by observation. Such representations include conceptual information about previous experience (hence memory), about the context in which the action has to be performed (e.g., danger, competition), up to its moral implications (if any).

The study of visuomotor behavior already reveals that even simple goal-directed movements are likely to be represented by the agent prior to their execution. Consider, for example, the action of grasping with the right hand a horizontally placed rod. Prior to his movement, the subject receives an instruction about what to do after the rod has been grasped: the instruction is (according to trials) either to place the right end or the left end of the rod on a stool. These instructions generate a highly consistent behavior. When the instruction is to place the right end of the rod on the stool, the subject invariably uses an overhand grip; conversely, for placing the left end of the rod on the stool, the subject uses an underhand grip (Rosenbaum \& Jorgensen, 1992). This process of grip selection (a typical example of visually based decision) suggests that biomechanical constraints generated by grasping the object and rotating the wrist are encoded within the representation of the movement: it is easier to rotate the hand in the pronation direction than in the supination direction (see Stelmach, Castiello, \& Jeannerod, 1994). An alternative explanation for this behavior would be that the visual configuration of the rod and the stool simply affords a pronation movement which is directly executed without building a representation. This alternative can be ruled out. Indeed, the same categorical decision is observed in a situation where the action of moving the rod to the stool is imagined (or simulated) but not executed (Johnson, 2000). Thus, mentally simulated hand movements follow the same rules and obey the same constraints as their overtly executed counterparts.

This finding was first reported in Parsons' (1994) hand matching experiment. In this experiment, a subject is shown the image of a sample hand in its canonical orientation. Another hand (the test hand) is then briefly presented at a different orientation and/or in a different posture. The subject's task is to tell whether or not the laterality (right or left) of the test hand matches that of the sample hand. The time taken by the subject to give the response is found to be a function of the difference in orientation between the two hands. So far, this result is in line with the well-known mental rotation phenomenon described in mentally matching two $3 \mathrm{D}$ 
visual shapes (e.g., Shepard \& Metzler, 1971). Unlike a 3D visual shape, however, one cannot rotate one's own hand in any direction: instead, the rotation of one's hand to a given orientation has to follow biomechanically compatible trajectories. This constraint is reflected in the results of the above hand matching experiment, where the response time is also a function of the compatible trajectory of the test hand, as if the subject were actually rotating his own hand. Other experiments of the same vein and using the same methodology of mental chronometry have confirmed that mentally imagined movements follow the same regularities as those which have been described for executed movements, for example, simulated reaching follows Fitts' law (Decety \& Jeannerod, 1996; Sirigu et al., 1996).

Thus, visuomotor representations appear to have a relatively direct influence on motor mechanisms, i.e., those mechanisms involved in the execution of the represented movements. This hypothesis is supported by the results of experiments where brain activity is monitored during cognitive tasks such as making visually based decisions, forming motor images or remembering motor events. These experiments (Decety et al., 1994; Parsons et al., 1995; Nilsson et al., 2000; Johnson et al., 2002; Shubotz \& von Cramon, 2002) reveal that, in the absence of any movement or muscular activity, brain areas corresponding to motor areas are activated. At the cortical level, primary sensorimotor cortex and dorsal and ventral premotor cortex are activated as well as, subcortically, the lateral cerebellum and basal ganglia. The activated structures partly but consistently overlap those that are activated during actual motor performance of the same actions (Gérardin et al., 2000; see review in Jeannerod \& Frak, 1999). Not surprisingly, increased neural activity in motor areas observed during a simulated action (e.g., mental hand rotation) is not observed during mental rotation of visual shapes, which only affects visual areas (Kosslyn et al., 1998).

Visuomotor representations and their close connections to motor execution, however, are only one among the possible classes of visual representations built for acting on the world. Their role is also to feed in more complex representations, more remote from visual input but which include more conceptual content. The contents of representations that result from higher level pragmatic processing include contextual elements drawn from the situation in which the action is taking place, such as the precise function of the objects which are part of this action. Consider, for example, a skilled action using tools. Tools, as well as musical instruments or sport materials, are objects which cannot be characterized merely by their geometrical properties like size, shape or orientation. They have additional properties that cannot be detected unless one knows what the object is for and how to use it; yet, once they are known (by observation, training or verbal instructions), they do supervene upon the pure geometrical properties that are part of the non-conceptual content of more basic visuomotor representations. Thus, the use of tools, the practice of musical instruments or the use of sport materials require the construction of visual representations whose con- ceptual content results from the pragmatic processing of visual information. For example, being able to use a tool and/or a musical instrument depends upon observing the skilled actions performed by others.

Watching other people act is indeed a source of information about the meaning of their actions and, ultimately, about the contents of their mental states. Observation of an action (e.g., performed with a tool or a musical instrument) first provides clues about the technical aspects of that action, for learning and replicating it. But observing an action and understanding its goal may also provide information about the agent's intentions, desires and motives. The capacity to imitate (which seems so distinctly human) depends on the ability to form visual representations of others' observed actions. Some perceptual representations of object-oriented actions play a crucial role in learning how to use such things as tools or musical instruments. They contribute to understanding the agent's motor intentions. One's representations of others' object-oriented actions share many of the neural correlates of one's own visuomotor decisions or of one's own imagined actions and motor imagery. This fact lends support to the idea that covert action or mental simulation is at work as well in the preparation of one's own object-oriented actions as in the perception and understanding of others' object-oriented actions (Jeannerod, 2001).

One may also watch a conspecific act, not in the context of learning a skilled action, but in a different social and emotional context. One may, for example, either watch the arm and hand movements of a person engaged in a fist-fight for the purpose of learning how to fight or in the social context of witnessing the action of an aggressor inflicting pain on a victim. Such a perceptually based representation of another's action encodes a wealth of visual stimuli endowed with a social, not a motoric (or technical), significance. In this case, the observed "actions", which are directed towards conspecifics, not towards inanimate objects, may include facial expressions, eye movements and fixations, changes in posture, or gestures which are not directed to external objects or goals, but which have a social ostensive or demonstrative role. Whereas perceptually based representations of objectoriented actions contribute to determining and understanding the agent's motor intention, perceptually based representations of actions directed towards conspecifics contribute to determining and understanding the agent's social intention, i.e., the agent's intention to affect a conspecific behavior. We argue elsewhere that, in the human brain, the cortical network associated with the perception of human actions directed towards manipulable objects is distinct from that associated with the perception of human actions directed towards conspecifics (Jacob \& Jeannerod, 2003).

As we noted at the end of Section 2, the neuropsychological dissociation between visual agnosia and optic ataxia is confirmation of the distinction between the world-to-mind direction of fit of semantic processing and the hybrid direction of fit of pragmatic processing. On the basis of this double dissociation, Milner and Goodale (1995) hypothesized the fol- 
lowing duality between the dorsal and the ventral pathway. In their model, the dorsal pathway underlies the visuomotor transformation, i.e., the crude, fast and automatic transformation of information about visual attributes of objects into motor commands. By contrast, the ventral pathway underlies visual perception, i.e., the conscious identification and recognition of objects. Although this model does capture one of the most obvious divisions of labor between visual pathways, it may not be entirely accurate: its main problem is that it wrongly draws a contrast between two kinds of visual information processing located in the two pathways at different levels of complexity. Both semantic processing, which depends on the activity of brain areas in the ventral stream, and pragmatic processing, which depends on the activity of brain areas in the dorsal stream, give rise to representations at different levels, whose content can be more or less conceptualized. Non-epistemic perception stands to semantic processing as the visuomotor transformation stands to pragmatic processing. No conclusion can be drawn about the differences between the semantic and the pragmatic processing of visual inputs unless the levels of conceptual content of their respective outputs are matched.

By contraposition, a valid comparison can be made between higher level representations in both the semantic and the pragmatic systems of processing. Neuropsychology offers a wealth of clinical observations of patients whose higher level representations for visually goal-directed actions are altered and whose behavior could be compared with the behavior of patients with deficits in semantic processing. The difficulties met by these patients appear in situations where they have to use tools for achieving a task on a visual goal. Yet, their impairment is not limited to motor execution: they also typically fail in tasks like pantomiming an action without holding the tool, imitating an action performed by another agent, judging errors from incorrectly displayed actions or imagining an action (motor imagery) (Clark et al., 1994; Sirigu et al., 1995; Ochipa et al., 1997; Goldenberg, Hartmann, \& Schlott, 2003). Such impairments in representing actions do not result from a general difficulty in visual recognition: Sirigu and Duhamel (2001) report the cases of two patients whose visual impairments in visual recognition tasks and in motor representations were dissociated. One patient with a left parietal lesion with ideomotor apraxia was unable to perform motor imagery tasks but had normal scores in visual imagery tasks. Conversely, another patient with agnosia for faces and visual objects had no visual imagery but normal motor imagery. Similarly, Tomasino, Rumiati, and Umilta (2002) report the case of one patient with ideomotor apraxia with a left parietal lesion, who was unable to perform the motor mental imagery task involving hand rotation, whereas he was still able to mentally rotate other visual stimuli.

The clinical observations quoted above stress the role of the parietal cortex in monitoring motor representations. Patients' impairments are produced by parietal lesions located in the angular and supramarginal gyri (the inferior parietal lobule), i.e., more anterior and ventral than those which produce a visuomotor impairment like optic ataxia. Furthermore, when the lesion is unilateral, it is more often localized in the left hemisphere, a lesional lateralization which is irrelevant to optic ataxia. Indeed, apraxic patients with a lesion of the left inferior parietal lobule have no basic visuomotor impairment: they can correctly reach and grasp objects. Rather, they are impaired in the recognition of tools and in the recognition of actions involving the use of tools. They cannot pantomime actions involving the use of an imaginary tool, nor can they recognize pantomimes executed by others. According to Glover's (in press) recent model, while the superior parietal lobule would be mainly involved in the on-line automatic control of basic visually guided actions towards objects, the left inferior parietal lobule would be involved in the higher level intentional planning of more complex actions involving the retrieval of complex representations thought to be stored precisely in that region. This role of the parietal cortex in action planning becomes even more obvious in the representation of non-executed actions, e.g., in imagined actions or in observing actions performed by another agent. Neuroimaging experiments in normal subjects, some of which have been already mentioned earlier in this paper, show that, besides activating motor areas in common with execution, motor representation tasks consistently activate areas in the posterior parietal lobe (Decety et al., 1994; Grafton, Arbib, Fadiga, \& Rizzolatti, 1996). In their recent study, Johnson et al. (2002) made a distinction between two mental tasks involving non-executed hand action: grip selection-a simple implicit visuomotor representation - and cued motor preparation, a process which involves attending to one hand and planning a movement with that hand. Grip selection primarily activated a dorsal area of the contralateral parietal lobe, whereas motor preparation activated parietal areas within the left hemisphere. These results are consistent with the above clinical observations, which dissociate visuomotor impairments from impairments in higher level motor representations involved in planning.

The separation, both anatomical and functional, between low-level representations for visuomotor transformation and higher level representations for planning suggests that the function of the occipitoparietal, dorsal, pathway should be refined. The function of the occipitoparietal pathway as described in the monkey, which reaches parietal areas within the intraparietal sulcus and which is connected to premotor areas, is indeed the achievement of the visuomotor transformation. The role of information processing in this pathway is to prepare biomechanically compatible limb trajectories, to compute the speed of the limb movements towards the target, and to adjust the size of the grip and the number of fingers involved for grasping it. These operations are likely to be largely automatic, for the sake of speed and accuracy, although they may be influenced by top-down processing for adapting the movement to the current situation. These points are illustrated by the behavior of patient AT. This patient presented the typical symptoms of optic ataxia exemplified 
by difficulties during reach and grasp movements: targets presented in her peripheral visual field were misreached, the grip size no longer correlated with object size, the orientation of the opposition axis during grasping no longer correlated with object's orientation (Jeannerod, Decety, \& Michel, 1994; Milner, Paulignan, Dijkerman, Michel, \& Jeannerod, 1999). However, when presented with familiar objects instead of neutral targets, AT's grasping performance improved significantly. This effect was likely to be due to a top-down control of the visuomotor transformation, originating from higher order, still intact, representations.

By contrast, higher order motor representations with more conceptual content appear to be independent from (though connected with) visuomotor representations. The fact that they are preferentially affected by left-sided lesions indicates that they pertain to a distinct system, concentrated in the inferior parietal lobule. As already mentioned, neuroimaging experiments reveal that the regions of the supramarginal gyrus and of the angular gyrus in the inferior parietal lobule are activated during tasks involving cued motor planning (e.g., hand selection), motor preparation or mental motor imagery. More recent investigations also found a strong activation of the inferior parietal lobule in tasks involving recognition of one's own actions as opposed to actions performed by another self (Ruby \& Decéty, 2001; Farrer et al., 2003).

\section{The quasi-conceptual content of the representation of spatial relations}

To visually represent an object is to represent it in space. One may think about objects that are or that are not in space (e.g., numbers or characters of fiction). But one cannot see objects that are not in space. There are at least three different ways in which one can represent (perhaps non-consciously) the spatial position of an object. All visual processing starts with visual information reaching the retina. So the spatial position of an object is first of all represented in retino-centered coordinates. For the purpose of reaching and grasping an object, however, the agent must form a representation of its spatial position in egocentric coordinates (i.e., centered on the axis of his body). In a perceptual task, the spatial position of an object relative to some other neighboring object is represented in allocentric coordinates (i.e., centered on some neighboring object). Thus, part of the duality between the semantic processing and the pragmatic processing of a visually presented object derives from the fact that the spatial position of an object can either be coded in allocentric or in egocentric coordinates. Representing the spatial position of an object in egocentric coordinates is required for reaching and grasping it. As we shall presently argue, representing the spatial position of an object in allocentric coordinates (thus representing its spatial relation to at least one other object present in the visual array) is required for full perceptual awareness of the object's other visual attributes.
Consider first experiments by Pylyshyn (2000a, 2000b) about so-called 'multiple object-tracking' (MOT) in normal human adults. First, subjects are shown eight identical circles at rest on a screen, four of which flicker briefly. Secondly, subjects see the eight circles move randomly on the screen for about $10 \mathrm{~s}$. Thirdly, they are asked to keep track of the four circles that initially flickered. Normal human adults can keep track of four-to-five such distinct objects (or proto-objects). Now comes the important observation: Pylyshyn reports that subjects fail to notice changes in the colors and shapes of the proto-objects that they tracked by their relative locations. We call 'proto-objects' the circles whose relative motions and positions normal adult subjects manage to track, precisely because such visual features as their shapes and colors are immaterial to their identity in the course of the task. The perceptual ability to visually represent the relative motions and locations of proto-objects is impervious to changes of colors and shapes. This ability clearly belongs to semantic processing, not to pragmatic processing. Engaging in a MOT experiment though seems almost like a cognitive task of spatial reasoning with little or none of the typical phenomenology of visual perception.

Neuropsychological studies of patients show that lesions in the dorsal pathway also frequently produce visuospatial impairments. Patients with lesions affecting the posterior parietal areas, usually in the right hemisphere, exhibit spatial disorientation: typically, these patients fail to determine the relative locations of objects: they are unable to describe a spatial trajectory and they cannot orient on a map. Lesions located in the right inferior parietal lobe typically produce unilateral spatial neglect. Unlike lesions in the superior parietal lobe, which produce optic ataxia and which can be on either side, lesions responsible for unilateral spatial neglect are generally located in the right hemisphere. Patients with unilateral spatial neglect are not perceptually aware of objects visually presented in their contralesional (i.e., left) hemispace. For example, when asked to mark line segments at different orientations, a neglect patient will systematically fail to mark the segments lying in her contralesional hemifield. If asked to bisect a horizontal line, she will exhibit a strong ipsilesional bias revealing neglect of the part of the line falling within her neglected hemispace.

Unlike blindsight patients whose primary visual cortex has been damaged, and to a lesser extent unlike visual form agnosic patients whose ventral stream has been impaired, neglect patients lack perceptual awareness on their affected side in spite of the fact that the visual pathway for processing the neglected visual information remains intact. Indeed, there is considerable evidence for covert processing of the neglected stimuli. For example, Marshall and Halligan (1994) showed neglect patient PS drawings of two houses located on top of each other, one of which displayed brightly colored flames on its left side. When asked to make an explicit comparison between the two houses, the patient could report no difference. When asked, however, which of the two houses she would rather live in, the patient 
pointed to the house without flames. This shows that the neglected stimuli are covertly processed in neglect patients even though this processing is not accompanied by perceptual awareness.

These disorders are clearly of a cognitive nature and correspond to a failure to build representations of spatial relationships between visual objects: one demonstration of this point is provided by the effects of posterior parietal lesions on a special kind of visual imagery (which we tentatively call spatial imagery), first described by Bisiach and Luzzatti (1978). These authors reported the case of a patient with left visuospatial neglect following a lesion of the right hemisphere, including the parietal lobe. When instructed to build a visual image of familiar surroundings and to describe the content of the image, the patient failed to describe objects located on the left side of his visual image. In other words, the patient seemed to have lost his topographical memory for that limited area of extrinsic space that was visually neglected in his spatial behavior.

This observation stresses the fact that what is usually called mental visual imagery should be divided into visual imagery of objects and visual spatial imagery. Whereas the former is involved in representing the visual attributes of objects (such as their color, texture, shape, contour and size) in the absence of retinal inputs, the latter is involved in representing the spatial positions and relations of what we called 'proto-objects' in the absence of retinal inputs. There is a double dissociation between impairments in the visual perception and recognition of objects (produced by inferotemporal lesions) and impairments in the representation of the spatial relationships between objects (produced by posterior parietal lesions). Levine, Warach, and Farah (1985) and Farah, Hammond, Levine, and Calvanio (1988) report similar dissociations between the visual imagery of objects and visual spatial imagery. Patients with impaired visual object recognition are also impaired for visual object imagery, whereas their ability for spatial imagery may be preserved. Conversely, patients with spatial disorientation are impaired in spatial imagery, but not in visual object imagery. Consider, for example, the associative agnosic patient LH described by Farah et al. Following a bilateral lesion of the occipitotemporal junction and of the inferotemporal cortex, this patient was deeply impaired in visual recognition for faces, animals, plants, food and many common objects. He was tested in a variety of tasks requiring visual imagery. He was asked about some of the characteristics of well-known objects that are rarely encoded in verbal memory and that require access to iconic memory such as: What is the color of a football?, Do beavers have long tails?, etc. LH was deficient in all these tasks. His deficit in visual object imagery, however, stood in contrast with his preserved ability for spatial imagery. Thus, LH was able to perform mental spatial tasks such as mental rotation of 3D letters or mental scanning.

The fact that visual spatial imagery was preserved in this patient has two important implications. First, it is congruent with the sparing of his parietal lobes, the role of which in spatial imagery was clearly demonstrated in normal subjects with neuroimaging (e.g., Kosslyn et al., 1998). Neuroimaging studies involving perceptual and visuospatial tasks (e.g., judgment of relative spatial location and orientation of two or more objects) also consistently show activation of relatively posterior and ventral parietal areas on the right side, in the fundus of the intraparietal sulcus (Haxby et al., 1994; Faillenot, Decety, \& Jeannerod, 1999) as well as in the area of the angular gyrus in the inferior parietal lobule (Köhler, Kapur, Moscovitch, Winocur, \& Houle, 1995). Second, this clinical fact demonstrates that visual processing in the dorsal pathway can build visual representations of the spatial relations among distinct proto-objects almost devoid of other visual attributes. In such representation with an abstract quasi-conceptual content and almost no pictorial content, proto-objects are individuated as relata of spatial relations. They become movable parts of visual scenes, events or pictures and their respective spatial arrangement can be subject to artistic composition by painters, designers or architects.

One important feature of unilateral neglect is that neglect patients are particularly vulnerable to the phenomenon of extinction: if presented with two competing stimuli in their contralesional left hemispace, they will typically fail to perceive the one further to their left. In other words, the stimulus located more towards the ipsilesional side will extinguish its competitor located more on the contralesional side. In one experiment, Driver and Vuilleumier (2001) presented a neglect patient with two conditions. In one condition, the stimulus was a Kanizsa white square whose subjective contours arose from the removal of the relevant quarter-segments from four black circles. In the other condition, the stimulus consisted of the four black circles in the same spatial positions, but the formation of the subjective contours of the Kanizsa white square was prevented by the fact that the four black circles were presented in their entirety. The patient extinguished most left-sided presentations of the stimulus in bilateral trials when the full presentation of the four black circles prevented the formation of the subjective contours of the Kanizsa white square. But extinction was much weaker when the patient could see the Kanizsa white square. In other words, the neglect patient found it easier to allocate her perceptual attention to one big object than to four competing smaller objects.

The importance of this finding lies in the fact that in neglect patients, the visual attributes of objects located in the neglected hemispace are still covertly processed by the relevant areas in the ventral pathway. But the patient remains unaware of the visual attributes of stimuli located in their neglected hemispace. By losing visual awareness of the relative spatial locations of objects in their neglected side, neglect patients also lose visual awareness of their other visual attributes of these objects. Loss of awareness of the spatial relations between objects (provoked by a lesion in the right inferior parietal lobe) produces loss of awareness of other visual attributes. But the dependency seems asymmetrical: 
loss of awareness of such visual properties of objects as their colors, shapes, sizes or orientations does not seem to lead to unawareness of the relative locations of objects.

On one hand, the claim that visual awareness of visual attributes (such as color, shape, size and orientation) asymmetrically depends on awareness of spatial relations among objects is consistent with the view that the representation of spatial relations among proto-objects has a quasi-conceptual character. On the other hand, this asymmetrical dependency fits with a conceptual analysis of what is the deep nature of visual perception. Visual awareness of the size, shape and orientation of one object consists in the perceptual comparison between its relative size, shape and orientation and those of neighboring objects. In other words, visual awareness must satisfy the constraint of contrastive identification (see Jacob \& Jeannerod, 2003). But comparative perceptual processing of the relative sizes, shapes and orientations of two or more objects in turn presupposes the representation of their relative spatial positions in some allocentric frame of reference and the possibility to mentally manipulate this representation.

Besides the case of neglect, further arguments in favor of the asymmetrical dependency of visual awareness of object identification upon awareness of spatial relations can be drawn from the observation of patients presenting other types of visuospatial disorders. Indeed, the processing of the spatial orientation of an object may interfere with the visual recognition and/or identification of that object. Warrington and Taylor (1973) presented right parietal brain-lesioned patients with photographs of common objects (e.g., a basket) taken from a non-conventional (or non-canonical) point of view. The patients failed to recognize these objects, although they had no problem recognizing the same objects when presented in a canonical view. Thus, the inability to mentally manipulate spatial relationships of visual objects (e.g., by mental rotation) might be responsible for the recognition impairment. Another related condition is dorsal simultagnosia. Typically, a dorsal simultagnosic patient will recognize most objects but will be unable to see more than one at a time, irrespective of their size. As a consequence of this condition, such patients cannot count objects; their description of complex scenes is slow and fragmentary; they behave like blind people when moving in a visual environment, groping for things and bumping into obstacles. Dorsal simultagnosia has been interpreted as a disorder of visual attention. Along with Posner, Walker, Friedrich, and Rafal (1984) hypothesis, Farah (1995) considers the possibility of a specific deficit in disengaging one's visual attention: in order to be able to engage one's visual attention onto a new stimulus, one must first disengage one's visual attention from its prior and/or current location. Parietal lobes would play a critical role in this attentional mechanism. People with a bilateral parietal lesion should thus present a 'sticky' attention on the current object without the possibility to shift to another one and, by way of consequence, without the possibility to build coherent spatial relationships between them.

\section{Conclusion}

Visual cognition appears to be far more complex than previously suggested by the current models opposing either visual object perception and space perception or perception and action. These models, which originated from the double dissociation paradigm, attempted to match a given aspect of visual function onto a given anatomical subdivision of the cortical visual system. The double dissociation paradigm, however, appears to be of a limited value when the number of the terms of the dissociation is greater than two. Clearly, according to the review above, there are more than two kinds of human visual representations and more than two-visual systems in the human brain.

In this paper, we have argued that no valid comparison between visual representations can arise unless provision is made for three critical properties: their direction of fit, their direction of causation and the level of their cognitive or conceptual content. The cognitive (or conceptual) content in turn is a function of the level of processing. Representations arising from earlier stages of processing-whether percepts or visuomotor representations-have very little or no conceptual content. Elementary visual percepts, for example, arise from the automatic stage of semantic processing whereby basic visual attributes of an object are assembled and bound together. Low-level visuomotor representations of targets of prehension result from the automatic process of the visuomotor transformation. Whether they result from semantic or from pragmatic processing, higher order representations get their conceptual content from the connections between visual cognition and other parts of the human cognitive system (such as the planning of action and semantic memory). They often proceed under conscious control. The two other critical properties of visual representations are their mind/world direction of fit and their mind/world direction of causation. The output of the semantic processing of visual inputs has a full mind-to-world direction of fit and a full world-to-mind direction of causation: on one hand, it visually registers the way the world is, on the other hand, it is caused by what it represents. The output of the pragmatic processing of visual inputs has both a hybrid direction of fit and a hybrid direction of causation: it yields information for the benefit of intentions, which clearly have both a world-to-mind direction of fit and a mind-to-world direction of causation. An intention is both the representation of a goal and a cause of the transformation of a goal into a fact. This two-fold distinction segregates representations specialized for perception from those specialized for action.

Since it penetrates deeply into visual knowledge of the world, visual perception cannot be limited to selecting an object from its surroundings, identifying it and giving it meaning. Semantic processing of visual inputs also implies comparison, which in turn requires that several objects be simultaneously represented and analyzed: hence, object perception in turn presupposes the representation of spatial relationships among two or more objects in a coordinate system indepen-
994 
dent from the perceiver. Spatial relationships in themselves carry cues for attributing meaning to an object, so that their processing is actually part of semantic processing of visual information. Thus, one has to consider that perception itself is actually distributed over the two classical pathways of the human visual system: the ventral and the dorsal cortical pathways. An intact (right) inferior parietal lobule is thus required for coding spatial relationships among objects in an allocentric frame of reference, which is itself part of the general process of semantic processing.

The above considerations about the role of the dorsal pathway in visual perception raise the point of the several functions of the human parietal lobe. Obviously, this brain region cannot be considered as a unitary entity with a single function. The superior parietal lobule carries visuomotor processing, a non-lateralized process common to monkey and man. The right inferior parietal lobule contributes to the perception of spatial relationships, a process with a mind-to-world direction of fit and a world-to-mind direction of causation. Finally, the left inferior parietal lobule contributes to still another type of representation, related to visually goal-directed action, i.e., with both a hybrid direction of fit and a hybrid direction of causation. The latter two processes are unique to man.

The identification of higher level motor representations as part of visual cognition-a critical aspect of pragmatic processing which has been one of the major themes of this paper-sheds new light on the relations between perception and action. As one moves from the automatic visuomotor transformation to the planning and execution of more complex actions involving the use and manipulation of tools, the distinction between action and perception loses much of its significance. On one hand, the ability to perform actions by using cultural tools and to pantomime the use of imaginary tools requires the storage of, and the ability to retrieve, schemas for tool manipulation. Such schemas are in turn formed on the basis of the observation of the actions of others. Thus, the ability to perform complex actions with tools relies on visual perception. On the other hand, schemas for the manipulation of tools are not only necessary for the appropriate actual or pantomimed use of tools but also for extracting the meaning of actions involving the use of tools by other agents. Not only is what one can do shaped by what one perceives, but also conversely what one can do shapes what one can perceive.

Not all perceived human actions are object-oriented actions. Nor are all human actions visually processed for the purpose of learning how to prepare and execute an action. Many human actions are directed towards conspecifics and many such actions are visually processed for the purpose of understanding their social and emotional significance. The processing of human actions directed towards conspecifics also gives rise to representations with various levels of conceptual content. At the lowest level of conceptual content is the visual perception of so-called "biological motion". Then at various higher levels of visual processing are perceptual representations of emotions carried by facial movements and

expressions. They in turn provide visual information to the 1104 human mindreading system (in Baron-Cohen's, 1995 terms), 1105 which underlies the attribution and recognition of mental 1106 states to others and to oneself.

\section{Uncited references}

Farah (1990) and Mountcastle, Lynch, Georgopoulos, Sakata, and Acuna (1975).

1109 1110

\section{References}

Anscombe, G. E. (1957). Intention. Oxford: Blackwell.

Baron-Cohen, S. (1995). Mindblindness, an essay on autism and theory of mind. Cambridge, MA: MIT Press.

Berhmann, M., Winocur, G., \& Moscovitch, M. (1992). Dissociation between mental imagery and object recognition in a brain-damaged patient. Nature, 359, 636-637.

Bisiach, E., \& Luzzatti, C. (1978). Unilateral neglect of representational space. Cortex, 14, 129-133.

Bridgeman, B., Kirsch, M., \& Sperling, A. (1981). Segregation of cognitive and motor aspects of visual function using induced motion. Perception and Psychophysics, 29, 336-342.

Clark, M. A., Merians, A. S., Kothari, A., Poizner, H., Macauley, B., Rothi, L. J. G., et al. (1994). Spatial planning deficits in limb apraxia. Brain, 117, 1093-1106.

Decety, J., \& Jeannerod, M. (1996). Fitts' law in mentally simulated movements. Behavioral Brain Research, 72, 127-134.

Decety, J., Perani, D., Jeannerod, M., Bettinardi, V., Tadary, B., Woods, R., et al. (1994). Mapping motor representations with PET. Nature, $371,600-602$.

Dretske, F. (1969). Seeing and knowing. Chicago: Chicago University Press.

Dretske, F. (1978). Simple seeing. In F. Dretske (Ed.), Perception, knowledge and belief. Cambridge: Cambridge University Press.

Driver, J., \& Vuilleumier. (2001). Perceptual awareness and its loss to unilateral neglect and extinction. In S. Dehaene \& L. Naccache (Eds.), The cognitive neuroscience of consciousness. Cambridge, MA: MIT Press.

Faillenot, I., Decety, J., \& Jeannerod, M. (1999). Human brain activity related to the perception of spatial features of objects. Neuroimage, 10, 114-124.

Farah, M. J. (1990). Visual agnosia. Disorders of object recognition and what they tell us about normal vision. Cambridge, MA: MIT Press.

Farah, M. J. (1995). Current issues in the neuropsychology of image generation. Neuropsychologia, 33, 1455-1471.

Farah, M. J., Hammond, K. M., Levine, D. M., \& Calvanio, R. (1988). Visual and spatial mental imagery. Dissociable systems of representation. Cognitive Psychology, 20, 439-462.

Farrer, C., Franck, N., Georgieff, N., Frith, C. D., Decety, J., \& Jeannerod, M. (2003). Modulating the experience of agency: A PET study. Neuroimage, 18, 324-333.

Gérardin, E., Sirigu, A., Lehericy, S., Poline, J. B., Gaymard, B., Marsault, C., et al. (2000). Partially overlapping neural networks for real and imagined hand movements. Cerebral Cortex, 10, 1093-1104.

Glover, S. Separate visual representations in the planning and control of action. Behavioral and Brain Sciences, in press.

Goldenberg, G., Hartmann, K., \& Schlott, I. (2003). Defective pantomime of object use in left brain damage: Apraxia or asymbolia? Neuropsychologia, 41, 1565-1573.

Goldenberg, G., Müllbacher, W., \& Nowak, A. (1995). Imagery without perception. A case study of ansognosia for cortical blindness. $\mathrm{Neu}$ ropsychologia, 33, 1373-1382. 
Goodale, M. A., Milner, A. D., Jakobson, L. S., \& Carey, D. P. (1991). A neurological dissociation between perceiving objects and grasping them. Nature, 349, 154-156.

Grafton, S. T., Arbib, M. A., Fadiga, L., \& Rizzolatti, G. (1996). Localization of grasp representations in humans by PET: 2. Observation compared with imagination. Experimental Brain Research, 112, 103-111.

Haxby, J. V., Horwitz, B., Ungerleider, L. G., Maisog, J. M., Pietrini, P., \& Grady, C. L. (1994). The functional organization of human extrastriate cortex. A PET-rCBF study of selective attention to faces and locations. Journal of Neurosciences, 14, 6336-6353.

Ingle, D. J. (1973). Two visual systems in the frog. Science, 181, 1053-1055.

Jacob, P., \& Jeannerod, M. (2003). Ways of seeing. The scope and limits of visual cognition. Oxford: Oxford University Press.

Jeannerod, M. (1986). The formation of finger grip during prehension. A cortically mediated visuomotor pattern. Behavioural Brain Research, 19, 99-116.

Jeannerod, M. (1997). The cognitive neuroscience of action. Oxford: Blackwell.

Jeannerod, M. (2001). Neural simulation of action: A unifying mechanism for motor cognition. Neuroimage, 14, S103-S109.

Jeannerod, M., \& Frak, V. G. (1999). Mental simulation of action in human subjects. Current Opinions in Neurobiology, 9, 735-739.

Jeannerod, M., Decety, J., \& Michel, F. (1994). Impairement of grasping movements following a bilateral posterior parietal lesion. Neuropsychologia, 32, 369-380.

Johnson, S. H. (2000). Thinking ahead: The case of motor imagery in prospective judgements of prehension. Cognition, 74, 33-70.

Johnson, S. H., Rotte, M., Gafton, S. T., Hinrichs, H., Gazzaniga, M. S., \& Heinze, H. J. (2002). Selective activation of a parietofrontal circuit during implicitly imagined prehension. Neuroimage, 17, 1693-1704.

Köhler, S., Kapur, K., Moscovitch, M., Winocur, G., \& Houle, S. (1995). Dissociation of pathways for object and spatial vision. A PET study in humans. NeuroReport, 6, 1865-1868.

Kosslyn, S. M., Alpert, N. M., Thomson, W. L., Maljkovic, V., Wiese, S. B., Chabris, C. F., et al. (1993). Visual mental imagery activates topographically organized visual cortex: PET investigation. Journal of Cognitive Neuroscience, 5, 263-287.

Kosslyn, S. M., DiGirolamo, G. J., Thompson, W. L., \& Alpert, N. M. (1998). Mental rotation of objects versus hands: Neural mechanisms revealed by positron emission tomography. Psychophysiology, 35, 151-161.

Levine, D. N., Warach, J., \& Farah, M. (1985). Two visual systems in mental imagery: Dissociation of "what" and "where" in imagery disorders due to bilateral posterior cerebral lesions. Neurology, 35, 1010-1018.

Marshall, J., \& Halligan, P. W. (1994). The yin and yang of visuospatial neglect. A case study. Neuropsychologia, 32, 1037-1057.

Millikan, R. G. (1996). Pushmi-pullyu representations. In J. Tomberlin (Ed.), Philosophical perspectives: vol. IX. Atascadero, CA: Ridgeview Publishing.

Milner, A. D., \& Goodale, M. A. (1995). The visual brain in action. Oxford: Oxford University Press.

Milner, A. D., Paulignan, Y., Dijkerman, H. C., Michel, F., \& Jeannerod, M. (1999). A paradoxical improvement of misreaching in optic ataxia: New evidence for two separate neural systems for visual localization. Proceedings of the Royal Society of London. Series B, 266, 2225-2229.

Mountcastle, V. B., Lynch, J. C., Georgopoulos, A., Sakata, H., \& Acuna, C. (1975). Posterior parietal association cortex of the monkey: Com- mand functions for operations within extra-personal space. Journal of Neurophysiology, 38, 871-908.

Nilsson, L. -G., Nyberg, L., Klingberg, T., Aberg, C., Persson, J., \& Roland, P. E. (2000). Activity in motor areas while remembering action events. NeuroReport, 11, 2199-2201.

Ochipa, C., Rapcsack, S. Z., Maher, L. M., Rothi, L. J. G., Bowers, D., \& Heilman, K. M. (1997). Selective deficit of praxic imagery in ideomotor apraxia. Neurology, 49, 474-480.

Parsons, L. M. (1994). Temporal and kinematic properties of motor behavior reflected in mentally simulated action. Journal of Experimental Psychology. Human Perception and Performance, 20, 709730.

Parsons, L. M., Fox, P. T., Downs, J. H., Glass, T., Hirsch, T. B., Martin C. C., et al. (1995). Use of implicit motor imagery for visual shape discrimination as revealed by PET. Nature, 375, 54-58.

Pinker, S. (1985). Visual cognition: An introduction. In S. Pinker (Ed.), Visual cognition (pp. 1-65). Cambridge, MA: MIT Press.

Posner, M. I., Walker, J. A., Friedrich, F. J., \& Rafal, R. D. (1984) Effects of parietal lobe injury on covert orienting of visual attention. Journal of Neuroscience, 4, 1863-1874.

Pylyshyn, Z. (2000a). Situating vision in the world. Trends in Cognitive Sciences, 4(5), 197-207.

Pylyshyn, Z. (2000b). Visual indexes, preconceptual objects and situated vision. Cognition, 80, 127-158.

Rosenbaum, D. A., \& Jorgensen, M. J. (1992). Planning macroscopic aspects of motor control. Human Movement Science, 11, 61-69.

Ruby, P., \& Decéty, J. (2001). Effect of subjective perspective taking during simulation of action: A PET investigation of agency. Nature Neurosciences, 4, 546-550.

Schneider, G. E. (1969). Two visual systems. Science, 163, 895-902.

Searle, J. R. (1983). Intentionality, an essay in the philosophy of mind. Cambridge: Cambridge University Press.

Servos, P., \& Goodale, M. A. (1995). Preserved visual imagery in visua form agnosia. Neuropsychologia, 33, 1383-1394.

Shepard, R. N., \& Metzler, J. (1971). Mental rotation of three dimensional objects. Science, 171, 701-703.

Shubotz, R. I., \& von Cramon, Y. (2002). Predicting perceptual events activates corresponding motor schemes in lateral premotor cortex: An fMRI study. Neuroimage, 15, 787-796.

Sirigu, A., \& Duhamel, J. R. (2001). Motor and visual imagery as two complementary but neurally dissociable mental processes. Journal of Cognitive Neuroscience, 13, 910-919.

Sirigu, A., Duhamel, J. R., Cohen, L., Pillon, B., Dubois, B., \& Agid, Y. (1996). The mental representation of hand movements after parietal cortex damage. Science, 273, 1564-1568.

Sirigu, A., Cohen, L., Duhamel, J. R., Pillon, B., Dubois, B., \& Agid, Y. (1995). A selective impairment of hand posture for object utilization in apraxia. Cortex, 31, 41-55.

Stelmach, G. E., Castiello, U., \& Jeannerod, M. (1994). Orineting the finger opposition space during prehension movements. Journal of Motor Behavior, 26, 178-186.

Ungerleider, L., \& Mishkin, M. (1982). Two cortical visual systems. In D. J. Ingle, M. A. Goodale, \& R. J. W. Mansfield (Eds.), Analysis of visual behavior (pp. 549-586). Cambridge: MIT Press.

Ungerleider, L. G., \& Haxby, J. V. (1994). 'What' and 'where' in the human brain. Current Opinion in Neurobiology, 4, 157-165.

Tomasino, B., Rumiati, R. I., \& Umilta, C. A. (2002). Selective deficit of motor imagery as tapped by a left-right decision of visually presented hands. Brain and Cognition.

Warrington, E. K., \& Taylor, A. M. (1973). The contribution of right parietal lobe to object recognition. Cortex, 9, 152-164. 